Document downloaded from:

http://hdl.handle.net/10251/56919

This paper must be cited as:

Giner Maravilla, E.; Sabsabi, M.; Ródenas García, JJ.; Fuenmayor Fernández, FJ. (2014). Direction of crack propagation in a complete contact fretting-fatigue problem. International Journal of Fatigue. 58:172-180. doi:10.1016/j.ijfatigue.2013.03.001.

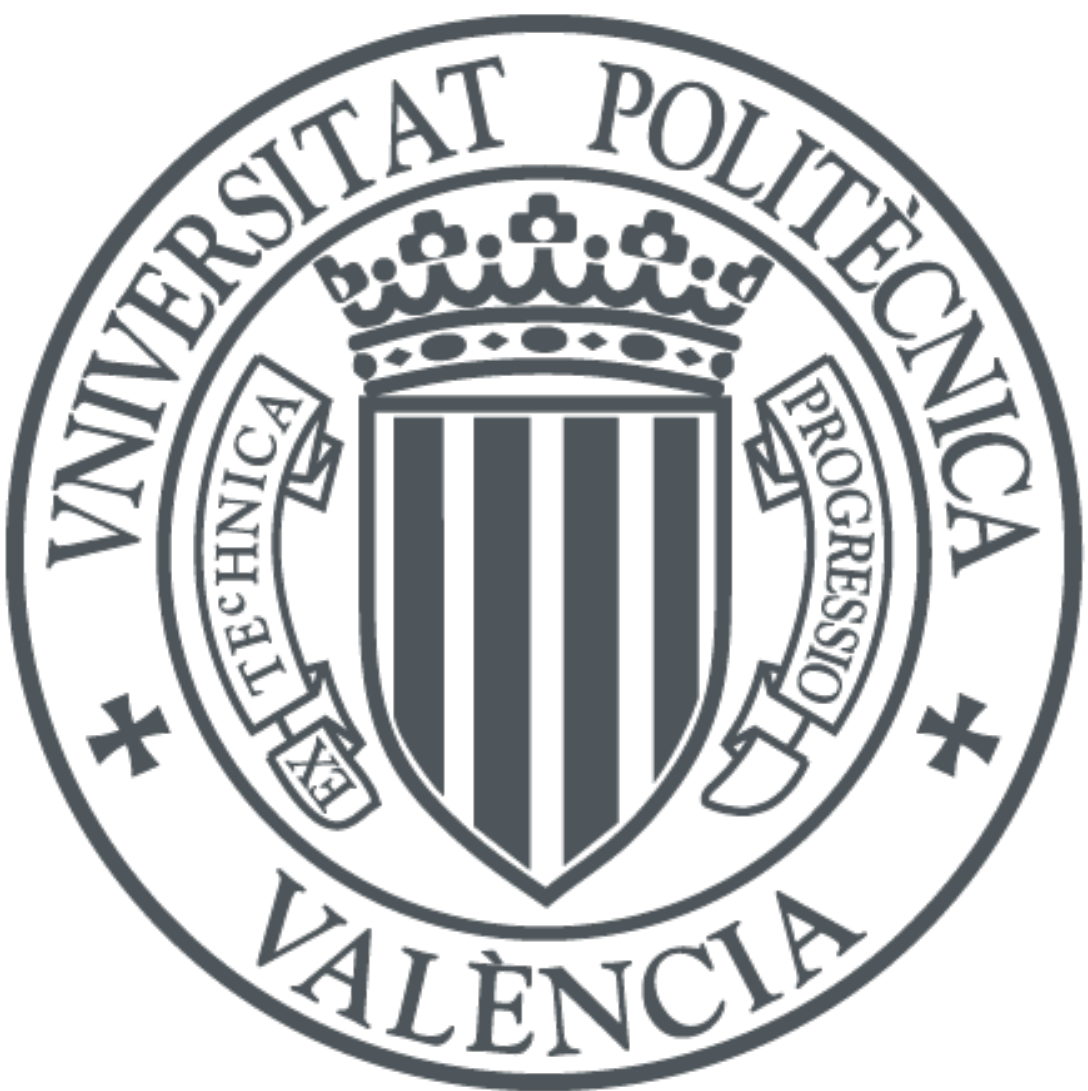

The final publication is available at

Copyright Elsevier

Additional Information 


\title{
Direction of crack propagation in a complete contact fretting-fatigue problem
}

\author{
Eugenio Giner*, Mohamad Sabsabi, Juan José Ródenas, \\ F. Javier Fuenmayor \\ Centro de Investigación de Tecnología de Vehículos - CITV, \\ Depto. de Ingeniería Mecánica y de Materiales, \\ Universitat Politècnica de València, Camino de Vera, 46022 Valencia, Spain.
}

\begin{abstract}
In this work, the orientation and propagation of a crack in a fretting fatigue problem is analyzed numerically and correlated experimentally. The analysis is performed using a 2D model of a complete-contact fretting problem, consisting of two square indenters pressed onto a specimen subjected to cyclic fatigue. For the simulation, we use the extended finite element method (X-FEM), allowing for crack face contact during the corresponding parts of the fatigue cycle. The problem is highly non-linear and nonproportional and an orientation criterion is introduced to predict the crack direction in each step of the crack growth simulation. It is shown that the proposed criterion predicts crack orientation directions that are in good agreement with those found experimentally, in contrast to the directions found by application of conventional orientation criteria used in LEFM, such as the MTS criterion.
\end{abstract}

Keywords: Crack paths; crack propagation; fretting fatigue; complete contact; extended finite elements.

\footnotetext{
*Corresponding author. Tel.: +34-96-3877007 ext. 76218; fax: +34-96-3877629.

Email address: eginerm@mcm.upv.es (Eugenio Giner)
} 


\section{Introduction}

Fretting fatigue problems involve two or more solids in contact that experience relative displacements of small amplitude. A general feature of fretting fatigue problems is that the contact region acts as a stress raiser causing crack initiation and subsequent crack propagation until the eventual failure of the component [1]. Due to the contact stresses, fretting fatigue problems are highly non-linear. In addition, a non-proportional evolution of the stress state often exists along the loading cycle. After initiation, crack propagation occurs in regions dominated by this complex stress state, which usually induces crack face contact and closure. It is also found that there is also a crack-contact interaction at the early stages of the crack growth, by which the contact stresses have an influence on the crack and, reciprocally, the crack presence alters the contact stress distribution $[2,3]$.

All these features make fretting fatigue problems difficult to analyze and numerical approaches often become necessary. In this work, we study a simple geometrical configuration under complete contact conditions, as sketched in Fig. 1: a specimen subjected to cyclic loading $\sigma_{\text {Bulk }}$ is pressed by two square indenters on two opposite sides through the action of a normal load $P$. In complete contacts, the contact area is independent of the load $P$ due to the abrupt change of the indenter geometry, in contrast to incomplete contacts, such as hertzian contacts. The abrupt change in geometry found in complete contacts also exhibit edges that behave as theoretically singular lines at the end of the contact area (four corner points in the 2D model of Fig. 1 that behave as singular points $[4,5])$. This stress state at the corner points leads to a rapid crack initiation stage as compared to incomplete fretting fatigue problems and a large percentage of the fatigue life is spent 
in the propagation stage $[1,6]$. As an indicative example, we estimated that the percentage of initiation life was mostly in the range $40-75 \%$ for the incomplete contacts analyzed in [3] (using a variable initiation length model), whereas for the complete contacts analyzed in [6], the initiation life was about $5-15 \%$ (using the same initiation model). Therefore, propagation clearly dominates in complete contacts and predicting the right propagation direction is essential to integrate crack growth laws in order to estimate the remaining life until failure. In this work, we assume that a small scale yielding condition prevails, both at the corner points [4] and at the crack tips, with a linear elastic material behaviour.

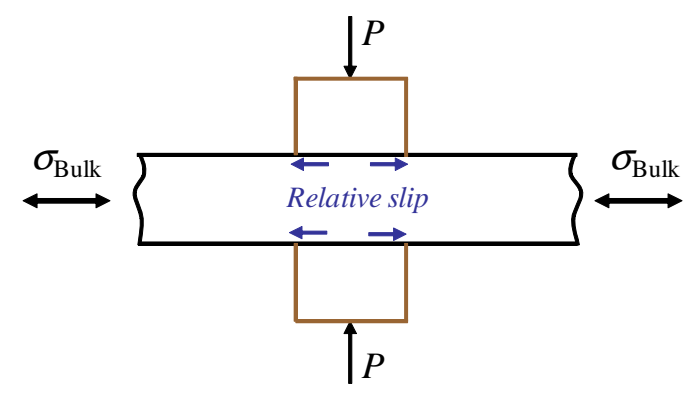

Figure 1: Sketch of the fretting fatigue problem under complete contact conditions.

The fretting configuration is analyzed both experimentally and numerically using the extended finite element method (X-FEM). The aim is to predict numerically the observed crack paths. The analysis of a $2 \mathrm{D}$ model of the physical tests is carried out using the X-FEM implementation developed by the authors [7-9]. The implementation is performed as a user's subroutine in the commercial code ABAQUS and can take into account crack face contacts along the loading cycle, which has been proved to be essential for the correct crack prediction. Several crack orientation criteria are reviewed in next section, together with the proposed approach based on 
the minimum shear stress range $\Delta \tau_{\min }$. In Section 3, the performed tests in aluminum 7075-T6 are described and micrographs of the experimental paths are provided. Finally, the numerical results in the last section show that the numerically predicted crack paths agree well with the experimental results.

\section{Crack orientation criteria for fretting fatigue problems}

\subsection{Review of existing criteria}

As commented above, fretting fatigue problems are characterized by the existence of multiaxial and high stress gradient zones subjected to mixed mode non-proportional loading [10], i.e. the directions of the principal stresses change along the cycle. The literature concerned with the direction of crack propagation in fatigue is vast and is not our aim to make a thorough review of existing criteria. Therefore, we will restrict this section to some of the works most relevant to the fretting fatigue conditions.

Following the classical description by Forsyth [11], usually two stages can be distinguished in the development of cracks: stage I for the initiation process and stage II for the subsequent propagation. Here we will assume that the crack is already initiated. In the initiation stage, cracks can exhibit a shallow angle with respect to the surface, called type 1 crack in stage I according to [12], which are dominated by the range of shear stresses $\Delta \tau$. This is not always the case and some cracks initiate with an angle much larger with respect to the surface (type 2 crack in stage I, according to the same reference [12]). This type 2 initiation cracks are controlled by the normal stress range $\Delta \sigma_{\mathrm{n}}$ where a high level of tensile stress exists. Type 2 initiation cracks are the case observed in our experimental tests with 
complete contact. It is important to note that we will concentrate only on the propagation phase (stage II, which is the dominating stage in complete contacts). Hence, we will assume that a type 2 crack already exists for the numerical model (i.e. stage I has already occurred).

It is well known $[10,13,14]$ that classical orientation criteria, such as the maximum tangential (circumferential or hoop) stress $\sigma_{\theta \theta}$ criterion (MTS) [15] or the minimum of the strain-energy-density factor $S$ [16] among others, are only valid for proportional loading. In the literature, several crack orientation criteria have been applied to fretting fatigue problems. For the analysis of fretting fatigue propagation (stage II) under non-proportional loading in an incomplete contact, Baietto-Dubourg and Lamacq [12] and Ribeaucourt et al. [17] considered the following criteria based on the work of Hourlier and Pineau [18]:

1. $\max \left(k_{\mathrm{I}}(\theta, t)\right)$ criterion: direction $\theta$ for which $k_{\mathrm{I}}$ attains its maximum along the cycle (absolute maximum in direction and time). Note that $k_{\mathrm{I}}$ is the mode I SIF associated with a virtual, infinitesimally small kinked segment emanating from the original crack with an angle $\theta$ (see Fig. 2).

2. $\max \left(\Delta k_{\mathrm{I}}(\theta)\right)$ criterion: direction $\theta$ for which $\Delta k_{\mathrm{I}}$ attains its maximum along the cycle.

3. $\max \left(\frac{\mathrm{d} a}{\mathrm{~d} N}(\theta)\right)$ criterion: direction $\theta$ for which $\frac{\mathrm{d} a}{\mathrm{~d} N}$ is maximum (maximum crack growth rate criterion).

The relations that enable to compute $k_{\mathrm{I}}, k_{\mathrm{II}}$ from $K_{\mathrm{I}}, K_{\mathrm{II}}$ are of the form:

$$
\begin{gathered}
k_{\mathrm{I}}(\theta)=K_{11}(\theta) K_{\mathrm{I}}+K_{12}(\theta) K_{\mathrm{II}} \\
k_{\mathrm{II}}(\theta)=K_{21}(\theta) K_{5}+K_{22}(\theta) K_{\mathrm{II}}
\end{gathered}
$$




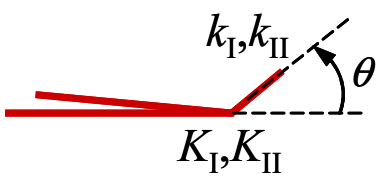

Figure 2: Prospective infinitesimal crack emanating from the original crack.

The angular functions $K_{i j}(\theta)$ are reported in [17] and are based on previous analysis by Amestoy et al. [19]. For the orientation angles usually found in practice, Eqs. (1) and the angular functions $K_{i j}(\theta)$ are essentially the same as the ones reported by Cotterell and Rice for proportional loading [20]. The relations proposed by Cotterell and Rice are simply the trigonometric functions of the crack tip singular fields expressed in polar stress components. They can also be found in fracture mechanics texts, such as [21]. The same equations are involved in the previously derived Nuismer criterion [22] for proportional loading. This criterion is based on the principle that the crack will propagate in such a way as to maximize the strain energy release rate $G$. For proportional loading, it can be shown [22] that it leads to the same result as the MTS criterion.

The above criteria by Hourlier et al. use the critical plane concept in the sense that the sought direction (plane) is the one in which the maximum magnitude is reached. The second of these criteria provided good results in [12] when applied to spherical (incomplete) contacts acting on prestressed specimens, although in [14] it is mentioned that this is not always the case. In [12], the first criterion did not correlate well with the experimental results and the third criterion was not applied due to the lack of experimental data (application of criterion 3 can be found in [17]). On the other hand, BaiettoDubourg and Lamacq [12] also proposed the following criterion: 
4. $\max \left(\Delta \sigma_{\theta \theta \text {,eff }}(\theta)\right)$ criterion: direction $\theta$ for which the effective range of the circumferential stress $\Delta \sigma_{\theta \theta}$ is maximum along the cycle (by effective, it is meant that $\sigma_{\theta \theta}=0$ when $\left.\sigma_{\theta \theta}<0\right)$.

In [12] this criterion led to similar results to criterion 2. The results in [12] emphasize the importance of evaluating the ranges $\Delta$ of the magnitude and not simply the maximum values: criteria 2 and 4 are both based on the concept of the maximum amplitude of crack opening. In this work, $\sigma_{\theta \theta}$ will also be denoted as $\sigma_{\mathrm{n}}$, indicating the normal stress to the prospective direction.

\subsection{The criterion of minimum shear stress range}

From the numerical analyses and for the geometric and loading configuration considered in this work, it is found that the crack remains closed during a large part of the loading cycle. The application of some of the criteria reviewed in the previous section did not lead to good predictions of the actual crack path as observed in the experimental tests performed. Consequently, it can be argued that the stress state existing under a crack face contact condition has an important influence to be considered. Assuming an elastic behaviour, the stress state under crack face contact conditions must be essentially controlled by $K_{\mathrm{II}}$, the only stress intensity factor that can exist for a totally closed crack in 2D.

The criterion proposed here is a generalization for non-proportional loading of the so-called "criterion of local symmetry" well established for proportional loading, see Cotterell and Rice [20]. The criterion of local symmetry states that the crack will propagate in the direction that $K_{\mathrm{II}}=0$. For nonproportional loading, the condition of $K_{\mathrm{II}}=0$ cannot be reached in general, 
and therefore, the proposed criterion seeks the angle for which the range $\Delta K_{\mathrm{II}}$ is minimized. This obviously reduces to the condition $K_{\mathrm{II}}=0$ when applied to proportional loading problems ${ }^{1}$.

In practice, computing $K_{\text {II }}$ values under crack face contact must include the effect of friction tractions on crack faces, as in [17, 23], which can be cumbersome and prone to inaccuracies when using domain and contour integrals. Instead, and equivalently, we will seek the angle for which the shear stress range $\Delta \tau$ at the crack tip is minimized. Shear stresses develop always in two orthogonal planes and there are two orthogonal planes on which $\Delta \tau$ is minimum. From these two potential crack growth directions, we choose the plane with the maximum $\Delta \sigma_{\mathrm{n}}$, because it will be the plane where less frictional energy is lost and there is more energy available for propagating the crack. This approach is in line with the principle that a crack will grow in the direction which maximizes the strain energy release rate $G[20,22]$.

As verified in the results presented in Section 6 , the $\Delta \tau_{\min }$ direction coincides with the direction of the maximum range of normal stress, $\max \left(\Delta \sigma_{\mathrm{n}}\right)$. This is due to the in-plane stress tensor transformation that yields both extremes in the same direction, although this may not be the general case. However, the direction predicted by the maximum range of the effective normal stress, $\max \left(\Delta \sigma_{\mathrm{n}, \mathrm{eff}}\right)$, does not lead to good results, at least in the problem studied here, despite the intuitive idea that only the positive normal stresses (effective) will govern the crack behaviour under an elastic material behaviour.

\footnotetext{
${ }^{1}$ We note in passing that, for proportional loading, the criteria of $K_{\mathrm{II}}=0$, Nuismer and MTS lead to the same result [20, 22].
} 
Fig. 3 sketches the convention used in the procedure. When applying the proposed criterion to the propagation stage, $\Delta \tau(\theta)$ is evaluated ahead the current crack tip. For each crack growth increment, stresses are evaluated ahead the crack tip and the prospective local direction $\theta$ is searched for which $\Delta \tau$ is minimum (see example of the estimation of the third increment direction in Fig. 3, left). In Section 6, the predicted angle is reported with respect to a fixed reference: $\beta$ is the predicted angle measured from the specimen surface. This way, a crack segment growing inwards (with respect to the indenter contact zone) has an angle $\beta>0$ and $\beta<0$ indicates a crack segment growing outwards.
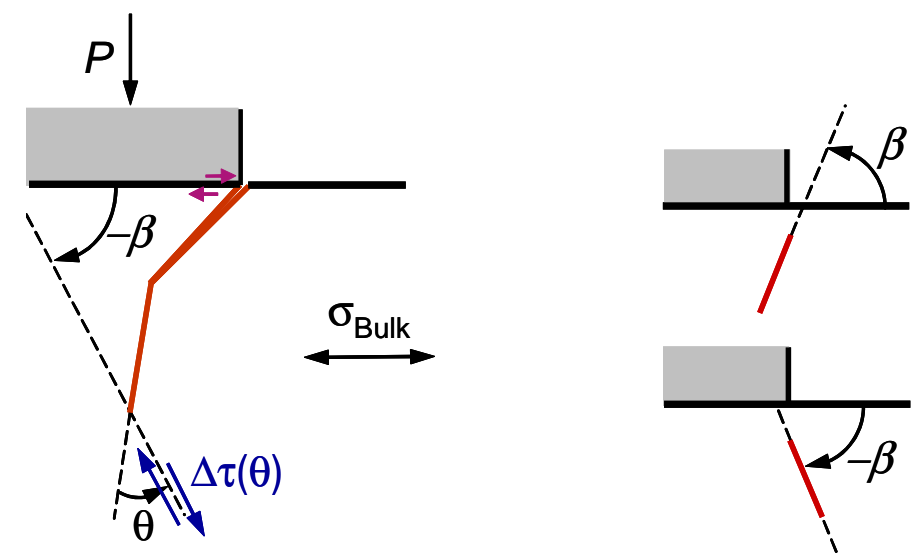

Figure 3: Application of the $\Delta \tau_{\text {min }}$ criterion to predict the third crack-growth increment direction. Sign convention for direction angles of a crack growth increment.

\section{Experimental tests}

In [6] we performed fretting fatigue tests with a square-ended indenter in a partial slip regime. The symmetrical relative slip produced by this complete contact configuration is sketched in Fig. 1. Tests were carried out with a uniaxial servo-hydraulic fatigue test machine with a load capacity of 
$100 \mathrm{kN}$. The assembly rig used to apply the normal load $P$ can be observed in Fig. 4. The cyclic bulk loading was performed at constant amplitude, stress ratio $R=-1$ at a frequency of $15 \mathrm{~Hz}$.

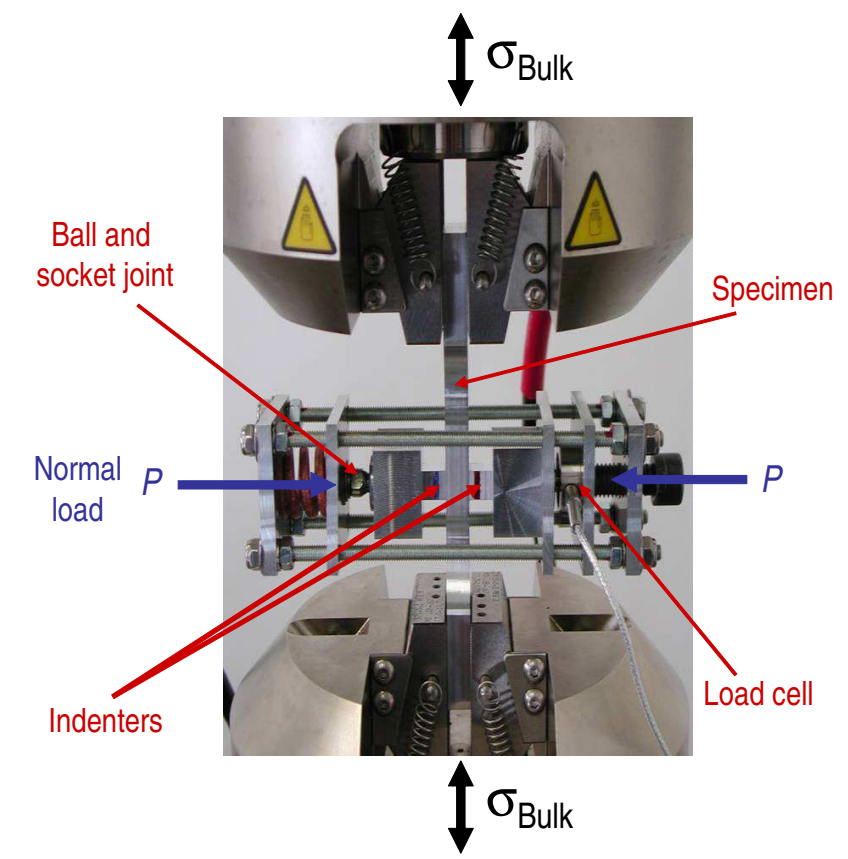

Figure 4: Complete contact testing rig, showing the contact elements.

Fifteen load combinations were analyzed in [6]. For this study, the following tests No. 1, 3, 5, 8, 11 and 15 were selected. The applied loads for each test and the experimentally registered number of cycles to failure are listed in Table 1. The nominal contact pressure is defined as $\sigma_{\mathrm{P}}=P /(2 c t)$, where $2 c$ is the contact width and $t$ the specimen and indenter thickness. The specimens are dog-bone shaped, with a rectangular section $t \times 2 B=5 \times 10 \mathrm{~mm}$, where $2 B$ is the width of the specimen (see also Fig. 7). The material for both specimens and indenters is an aluminum alloy 7075-T6, with a Young's modulus of 72 GPa and a Poisson's ratio of 
0.3. The indenters have a rectangular cross section of $t \times 2 c=5 \times 10 \mathrm{~mm}$, i.e. the same thickness as the specimens. Further details regarding the experimental tests and material characterization can be found in [6].

Table 1: Loads and experimental number of cycles to failure for the tests considered.

\begin{tabular}{ccccc}
\hline Test number & $P(\mathrm{kN})$ & $\sigma_{\mathrm{P}}(\mathrm{MPa})$ & $\sigma_{\text {Bulk }}(\mathrm{MPa})$ & $N_{f}$ (cycles) \\
\hline 1 & 2 & 40 & 110 & 105958 \\
3 & 8 & 160 & 110 & 82549 \\
5 & 4 & 80 & 130 & 47714 \\
8 & 4 & 80 & 150 & 32905 \\
11 & 4 & 80 & 170 & 27391 \\
15 & 8 & 160 & 190 & 8760 \\
\hline
\end{tabular}

After failure, specimens were ground on the plane of Fig. 1 in order to take micrographs of propagated cracks initiated at the corners of Fig. 1. Fig. 5 shows the micrographs for the propagation stage (the total length shown for the longer cracks in the picture is about $1.2 \mathrm{~mm}$ ). These are cracks that did not lead to final failure and that emanated from one of the four corners of Fig. 1. It can be observed that the crack paths are very similar for all of them: the crack grows inwards and deviates from the initiation angle $\beta \approx 60^{\circ}$ to about $\beta \approx 80^{\circ}$. Note that, despite the irregularities due to the local microstructure, experimental evidence shows that the growth trend is always inwards in this region.

\section{Numerical modelling with the extended finite element method}

In this work, the extended finite element method [24] is used to model the crack propagation. The main feature of the X-FEM is the enrichment of the FEM model with additional degrees of freedom (DOFs). These additional DOFs are associated with the nodes of the elements that are geometrically intersected by the crack location (called enriched nodes and 

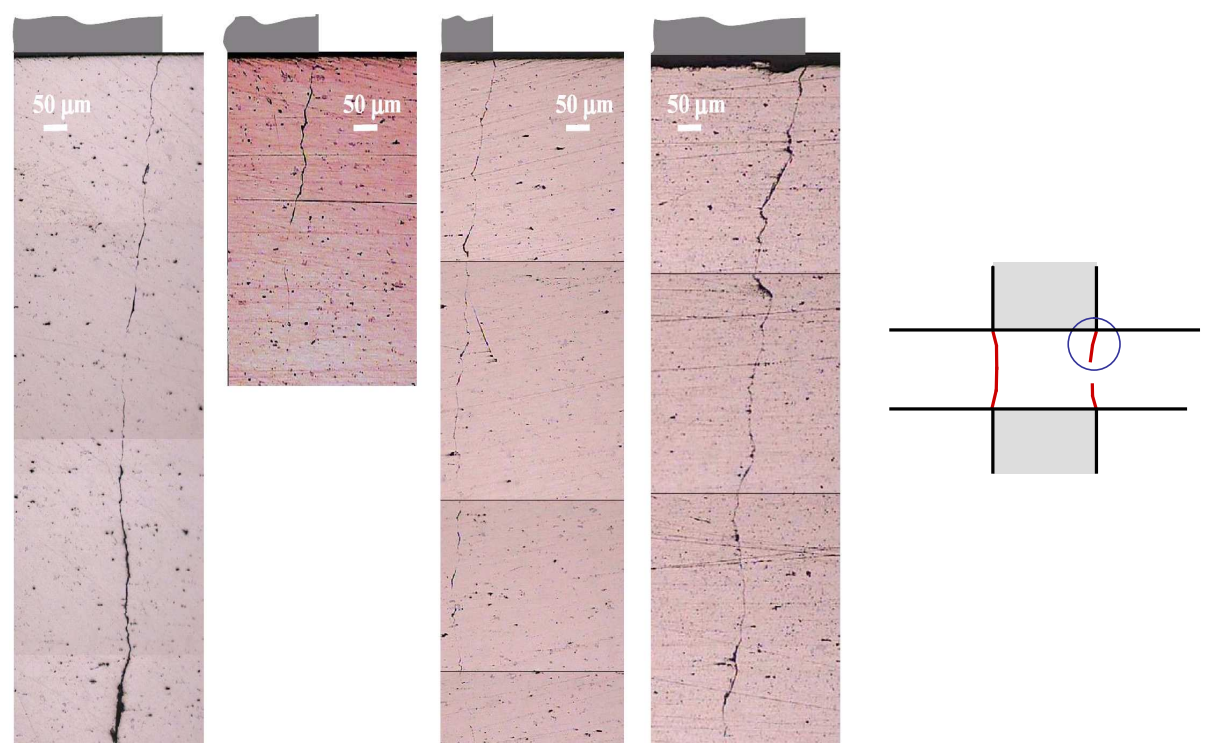

Figure 5: Micrographs for the propagation of non-failure cracks. Tests 1, 5, 8 and 15.

elements, respectively). Thus, the discontinuity is included in the numerical model without modifying the discretization. Fig. 6 shows a portion of a mesh where the enriched nodes are marked. Nodes located next to the crack faces (marked as circles in Fig. 6) are enriched with 2 additional DOFs (one for each direction of the domain space) to represent the physical displacement discontinuity by means of a Heaviside function $H(\mathbf{x})$. The Heaviside function can only take the values $H(\mathbf{x})= \pm 1$, depending on the relative position of the enriched node with respect to the crack face.

The X-FEM formulation allows for a further type of enrichment for those nodes that surround the crack-tip. These nodes (marked as squares in Fig. 6 are enriched with 8 additional DOFs: four crack-tip functions $F_{l}(\mathbf{x})$ times the two directions of the domain space. The crack-tip functions constitute the basis functions that represent the first term of the LEFM displacement field, and consequently, can reproduce the classical singular behaviour of 


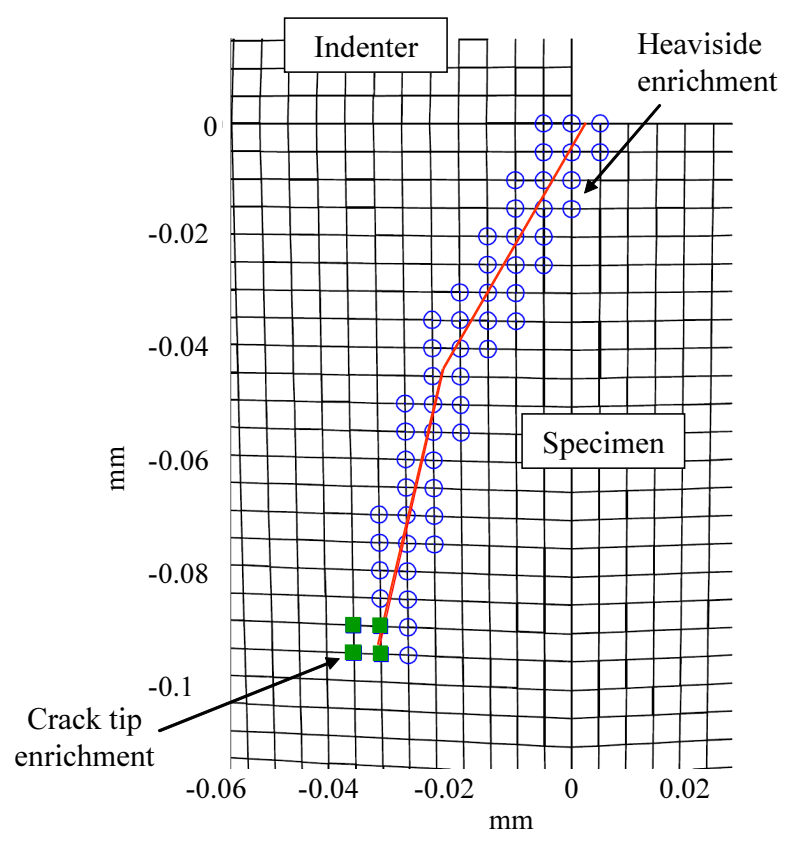

Figure 6: Enriched nodes in the X-FEM.

the LEFM strain and stress fields. These functions are given by [24]:

$$
\left[F_{l}(r, \theta), \quad l=1-4\right]=\left[\sqrt{r} \sin \frac{\theta}{2}, \sqrt{r} \cos \frac{\theta}{2}, \sqrt{r} \sin \frac{\theta}{2} \sin \theta, \sqrt{r} \cos \frac{\theta}{2} \sin \theta\right]
$$

where $r, \theta$ are polar coordinates defined with respect to the local reference system at the crack tip. Thus, for the 2D case, the extended finite element approximation to the displacements using the so-called "shifted" basis formulation at a point of the domain $\mathbf{x}$ is $[25,26]$ : 


$$
\begin{aligned}
\mathbf{u}_{\mathrm{xfem}}(\mathbf{x})=\sum_{i \in \mathcal{I}} N_{i}(\mathbf{x}) \mathbf{u}_{i}+\sum_{i \in \mathcal{J}} N_{i}(\mathbf{x})\left[H(\mathbf{x})-H\left(\mathbf{x}_{i}\right)\right] \mathbf{a}_{i}+ \\
+\sum_{i \in \mathcal{K}}\left[N_{i}(\mathbf{x}) \sum_{l=1}^{4}\left[F_{l}(\mathbf{x})-F_{l}\left(\mathbf{x}_{i}\right)\right] \mathbf{b}_{i l}\right]
\end{aligned}
$$

where $\mathcal{I}$ is the set of all nodes and the subsets $\mathcal{J}, \mathcal{K}$ contain the enriched nodes with either the Heaviside function $H(\mathbf{x})$ or with the crack-tip functions $F_{l}(\mathbf{x})$, respectively. $N_{i}(\mathbf{x}), \mathbf{u}_{i}$ are the standard shape functions and displacements of each node $i$, respectively, and $\mathbf{a}_{i}, \mathbf{b}_{i l}$ are the additional DOFs associated with the Heaviside function $H(\mathbf{x})$ and the crack-tip functions $F_{l}(\mathbf{x})$. Note that the introduction of the crack-tip functions enhances the quality of the calculated singular stress field in the vicinity of the crack tip. This is a further advantage of the X-FEM over the standard FEM [24].

In this work, we used the implementation proposed by the authors [7, 27], in which the X-FEM approach is integrated in ABAQUS by defining a user element that allows 12 DOFs/node. The combination of the powerful contact procedures available in ABAQUS with the X-FEM implementation has proved successful. The SIFs calculation can then be performed by means of the path or domain independent interaction integral (further details are given in [7]).

As is widely known, one to the major complications in the study of mixed mode fatigue crack growth is the frictional contact of the mating crack faces [28]. An essential feature of the current implementation is the capability of modelling crack face contact and closure with X-FEM. During 
the loading cycle, crack remains closed during a large part of the cycle (we recall that the loading stress ratio is $R=-1$ ). Therefore, the numerical model must capture the crack opening and closure, including crack face friction, because the stress fields in the vicinity of the crack tip change substantially during the cycle. Our analyses show that the consideration of crack closure is the only way to predict growth directions in good agreement with the experimental results. The numerical details related to the X-FEM implementation incorporating crack face contact with friction are beyond the scope of this work. The interested reader is referred to $[8,23]$. Recent works in the literature by Baietto, Gravouil et al. [29, 30] also apply X-FEM to fretting problems considering crack face contact.

\section{Description of the numerical model}

Due to symmetry conditions, a quarter 2D finite element model has been considered to represent the fretting fatigue tests, as shown in Fig 7. The rectangle $L \times B$ corresponds to the portion of the analyzed specimen and has a length of $L=4 B=20 \mathrm{~mm}$, the half length of the indenter $c$ is $5 \mathrm{~mm}$, and the distance between the contact plane and the point of the indenter at which loads are applied is $h=10 \mathrm{~mm}$. Four node, plane strain quadrilateral elements were used with a thickness $t=5 \mathrm{~mm}$. The smallest element size considered is $5 \mu \mathrm{m}$ at the right end of the contact zone.

The friction model assumed for the contact zone is a Coulomb model and the ABAQUS contact formulation based on Lagrange multipliers is used to model the contact between the indenter and the specimen. The friction coefficient is taken as $\mu=0.8$ [31]. The material behaviour is assumed linear elastic, despite the high stress concentration at the contact 

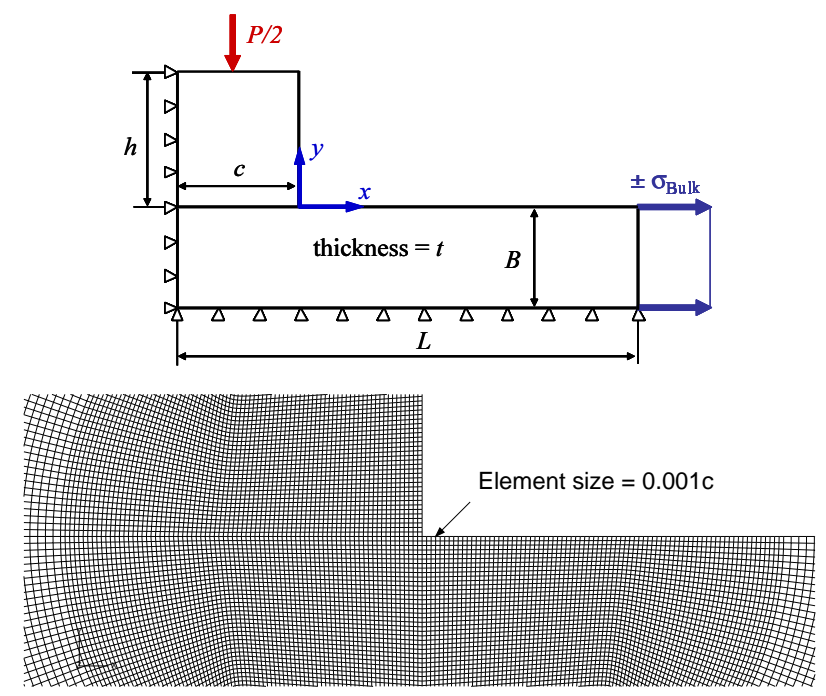

Figure 7: Model geometry and enlarged view of the FE discretization.

edge. The application of the linear regime is deemed valid, due to the very small edge radius of the indenter and the relative high yield stress of the aluminum alloy. As a consequence, the existing plasticity is very localized and a small scale yielding assumption can be applied, analogous to the small scale yielding assumption admitted in LEFM around the crack tip. This is confirmed by the observation of the tested specimens, which showed no macroscopic evidence of plasticity (see micrographs in Fig. 5 in which crack faces match very well each other and a view of the specimen contact surface in Fig. 7 of our previous work [6]).

The loading sequence is represented in Fig. 8, where six load steps have been considered in the analysis. Due to the non-linearity of the contact problem, loads were applied in sufficiently small time increments. In order to give a physical insight into the mechanics of the model under this loading, Fig. 9 shows enlarged views of the von Mises stress field at four instants of the loading sequence. For the sake of clarity, the X-FEM model in Fig. 9 


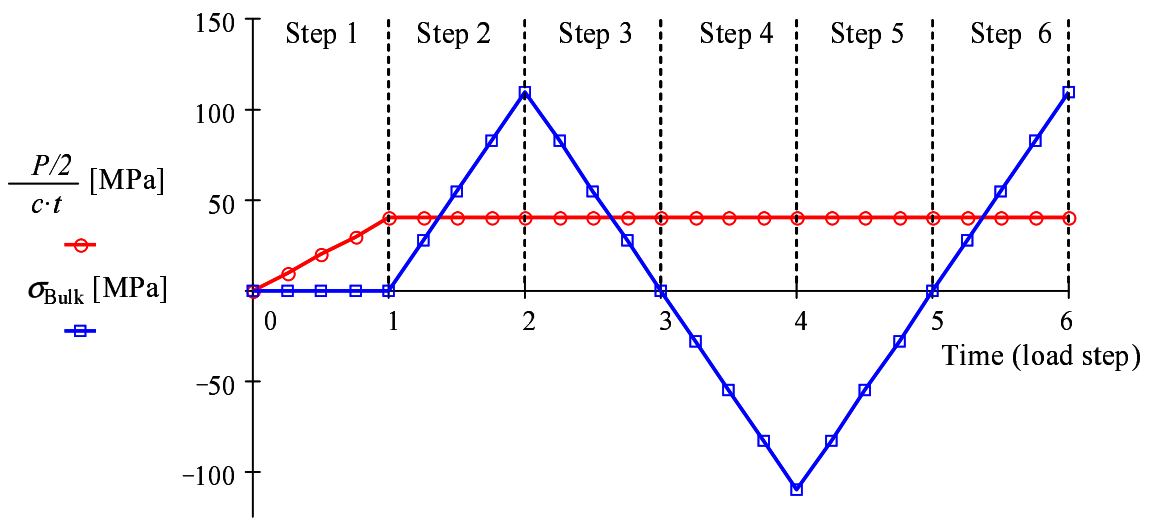

Figure 8: Loads applied to the numerical model for test 1.

represents a propagated crack after ten crack growth increments $\Delta a$, as explained in the following Section 6 .

At time $t=2.00$ the maximum $\sigma_{\text {Bulk }}$ is being applied, which produces a clear opening of the crack (displacements are magnified by $10 \times$ ). The expected von Mises stress field characteristic of a dominating mode I can be observed in the vicinity of the crack tip. The zone in grey between the open crack faces represents the deformation of the X-FEM enriched elements that introduce the mathematical discontinuity to model the crack face separation. When the bulk load is decreased $(t=2.75)$, mode I is reduced and the von Mises stress field indicates a clear mixed mode condition. Note that the vertical load due to the indenter is kept constant during the cycle and mode II increases its dominance over mode I as $\sigma_{\text {Bulk }}$ is reduced. At time $t=3.00$ crack face contact is produced and a mode II condition is present at the crack tip. Note also that kinks along the crack faces behave as local stress raisers when entering into contact with the opposing face. At time $t=4.00$ the bulk load is completely reversed and the load is transmitted through the crack faces. The end of the contact zone acts now as a strong 

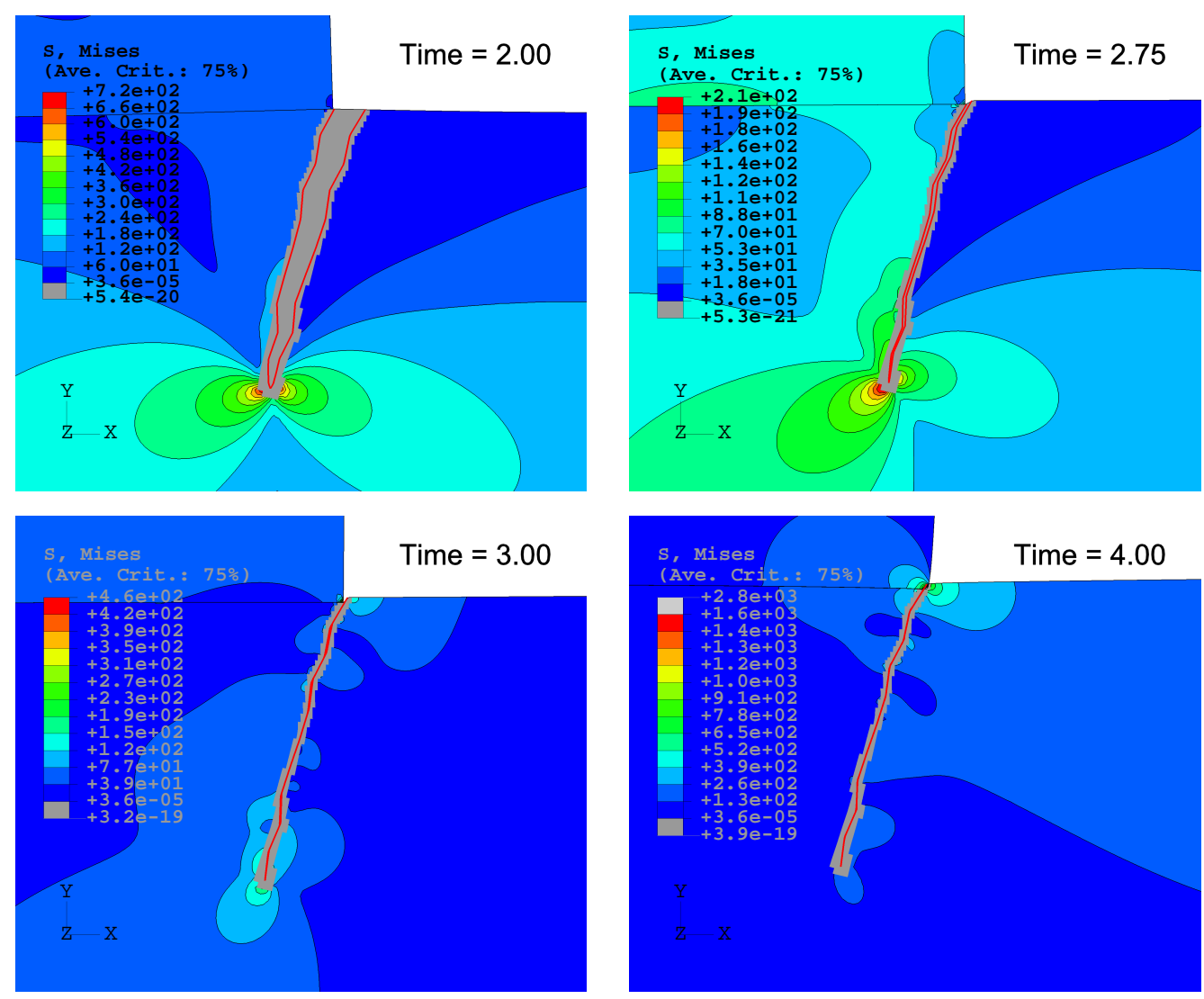

Figure 9: Enlarged view of the von Mises stress field at four instants of the loading sequence shown in Fig. 8. Displacements are magnified by a factor of $10 \times$.

stress raiser, as the specimen is compressed against the contact corner.

Results in the following section are presented for the load steps 5 and 6 (when "shakedown" of the numerical model response is produced). It has been verified that the stress states at $t=5$ and $t=6$ are very similar to those at $t=3$ and $t=2$, respectively.

\section{Crack growth simulation and comparison with experimental results}




\subsection{Model with no crack}

The first objective is to estimate the crack growth angle at the very early stages of propagation, i.e. for crack lengths of $50-75 \mu \mathrm{m}$. This implies that the crack is still small but significantly larger than the grain size of the material, which in our problem is about $35 \mu \mathrm{m}$ [27]. For the complete contact configuration of this work, the experimental evidence shows that the initiation crack is a type 2 crack, i.e. the initiation angle is not shallow [12]. To this aim, we used the numerical model of Fig. 7 prior to the presence of any crack, i.e. X-FEM is not used and a standard FE analysis is performed.

Crack initiation in the specimen is expected in the neighbourhood of the contact corner. Therefore, the stresses at the integration points of an element in this region were recorded along the cycle. A critical plane analysis was carried out to compute $\Delta \tau, \Delta \sigma_{\mathrm{n}}$ and $\Delta \sigma_{\mathrm{n} \text {,eff }}$ for different prospective angles $\beta$ and the results are plotted in Fig. 10. This figure shows the estimation of the initial direction for Test 1 . It can be observed that the criterion of $\Delta \tau_{\text {min }}$ yields two minima. Choosing the one with the highest $\Delta \sigma_{\mathrm{n}}$ a good prediction of the initiation angle is found $\left(\beta \approx 60^{\circ}\right)$. Note that the application of the criterion $4, \max \left(\Delta \sigma_{\mathrm{n}, \mathrm{eff}}\right)$, yields the estimation $\beta \approx 90^{\circ}$, which is not correct. This means that compressive part of the cycle cannot be disregarded when estimating the range of variation of $\sigma_{\mathrm{n}}$.

On the other hand, it is verified that $\max \left(\Delta \sigma_{\mathrm{n}}\right)$ leads to the same estimation as $\Delta \tau_{\min }$. However, the $\Delta \tau_{\min }$ criterion always gives a sharper indication of the predicted angle in comparison with $\max \left(\Delta \sigma_{\mathrm{n}}\right)$. The slopes around the points of $\Delta \tau_{\min }$ are greater than the slopes around the points of $\max \left(\Delta \sigma_{\mathrm{n}}\right)$, enabling a better estimation of the angle $\beta$. 


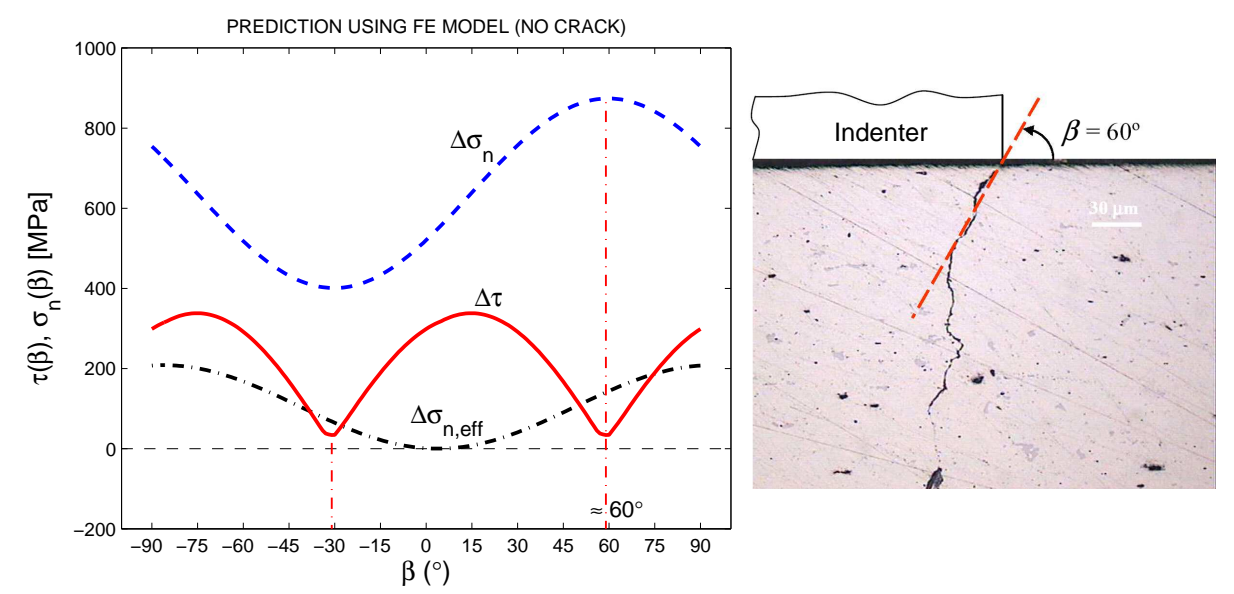

Figure 10: Predicted initial direction for a type II crack using the $\Delta \tau_{\min }$ criterion for test 1 . The analysis is performed with no crack, using standard FEM.

\subsection{Model with X-FEM crack}

Fig. 10 shows a good correlation with the experimental results at the first stages of the crack growth. Therefore an initial crack with angle $\beta=60^{\circ}$ is introduced in an X-FEM model. The length of this initial crack is taken as $a_{0}=50 \mu \mathrm{m}$ as discussed in [27]. This length $a_{0}$ is of the order of several grains for this material [9], a necessary condition for the applicability of the LEFM. The introduction of the initial crack through X-FEM presents the enormous advantage that it does not need remeshing to simulate the initial crack or any subsequent crack propagation, because the element sides do not need to conform to the crack faces. In addition, the numerical approximation of the stress fields in the vicinity of the crack tip is enhanced by the crack-tip enrichment functions.

For the application of the $\Delta \tau_{\min }$ criterion, the stresses at the integration points of the element ahead of the crack tip are recorded along the steps 5 and 6, i.e. in the range $t \in[4.0,6.0]$. Fig. 11 shows the variation of $\tau$ and $\sigma_{\mathrm{n}}$ with the prospective direction $\beta$ and for the different time increments 

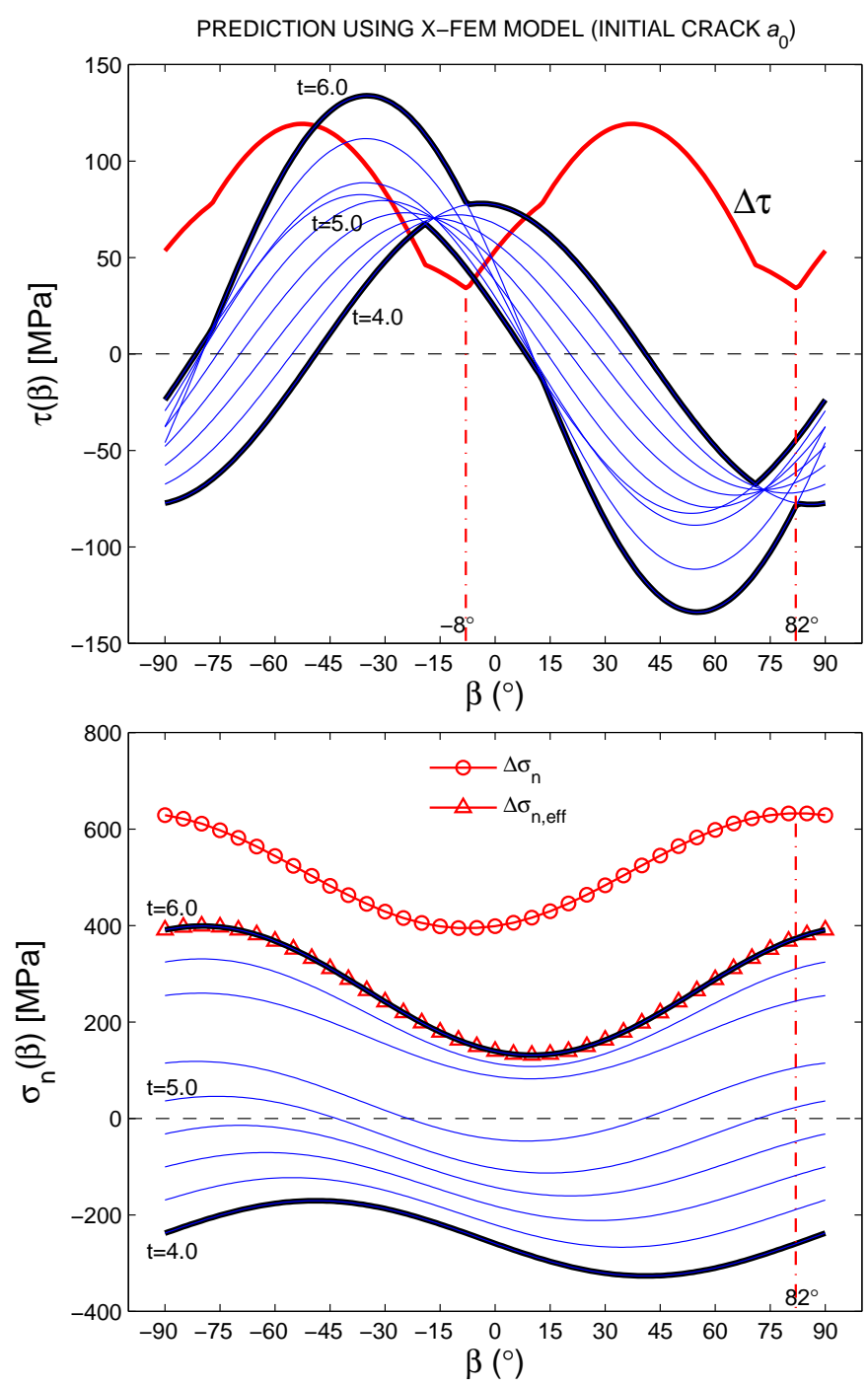

Figure 11: Prediction of crack growth direction ahead of the initial crack $a_{0}=50 \mu \mathrm{m}$, $\beta_{0}=60^{\circ}$ with the $\Delta \tau_{\min }$ criterion for test 1 . The crack is modelled using X-FEM.

$t \in[4.0,6.0]$. Note that the position of the maxima and minima for each time increment varies, which is indicative of the non-proportionality of the loading. Taking the maximum and minimum envelopes of the curves, the ranges $\Delta \tau$ and $\Delta \sigma_{\mathrm{n}}$ can be easily computed. The effective range $\Delta \sigma_{\mathrm{n}, \mathrm{eff}}$ is also provided. 
The predicted angle is chosen as the one that leads to $\Delta \tau_{\min }$ in combination with the greatest $\Delta \sigma_{\mathrm{n}}$. In this case, the predicted angle is $\beta=82^{\circ}$, which according to the convention of Fig. 3, indicates a slightly inwards direction, in good agreement with experimental evidence. Note that the criterion 4 based on $\max \left(\Delta \sigma_{\mathrm{n} \text {,eff }}\right)$ would predict an angle about $-80^{\circ}$, i.e. slightly outwards. This is incorrect due to ignoring the influence of the compressive part of the cycle. Application of the MTS criterion leads to a very similar wrong value owing to the same reasons, as can be seen in [27] for similar problems. Note also that $\Delta \tau$ at the angle of $\max \left(\Delta \sigma_{\mathrm{n}, \mathrm{eff}}\right)$ is substantially greater than $\Delta \tau_{\min }$.

The procedure is repeated adding new crack increments. The subsequent crack growth increments are taken as $\Delta a=50 \mu \mathrm{m}$, a value that it is recommended to be greater than four or five times the element size in the region analyzed. Fig. 12 shows the application of the $\Delta \tau_{\min }$ criterion after four crack growth increments, i.e. $a=a_{0}+4 \Delta a$. In this case, the direction of the next crack growth increment is predicted to be $\beta=70^{\circ}$, again pointing inwards beneath the indenter contact zone.

Fig. 13 plots the prediction for the propagation stage of Test 1 after six crack growth increments, $a=a_{0}+6 \Delta a$. The figure on the left represents the predicted propagation path with the proposed criterion $\Delta \tau_{\min }$ using $\mathrm{X}$ FEM and taking into account crack face contact as explained above. It is observed that the predicted path is in good agreement with the experimental observations. Note in Fig. 13 (right) that the simple application of the MTS criterion at the instant of maximum $\sigma_{\text {Bulk }}$, i.e. when the crack is fully open and disregarding what happens at the rest of the cycle, does not yield correct predictions of the crack path. 

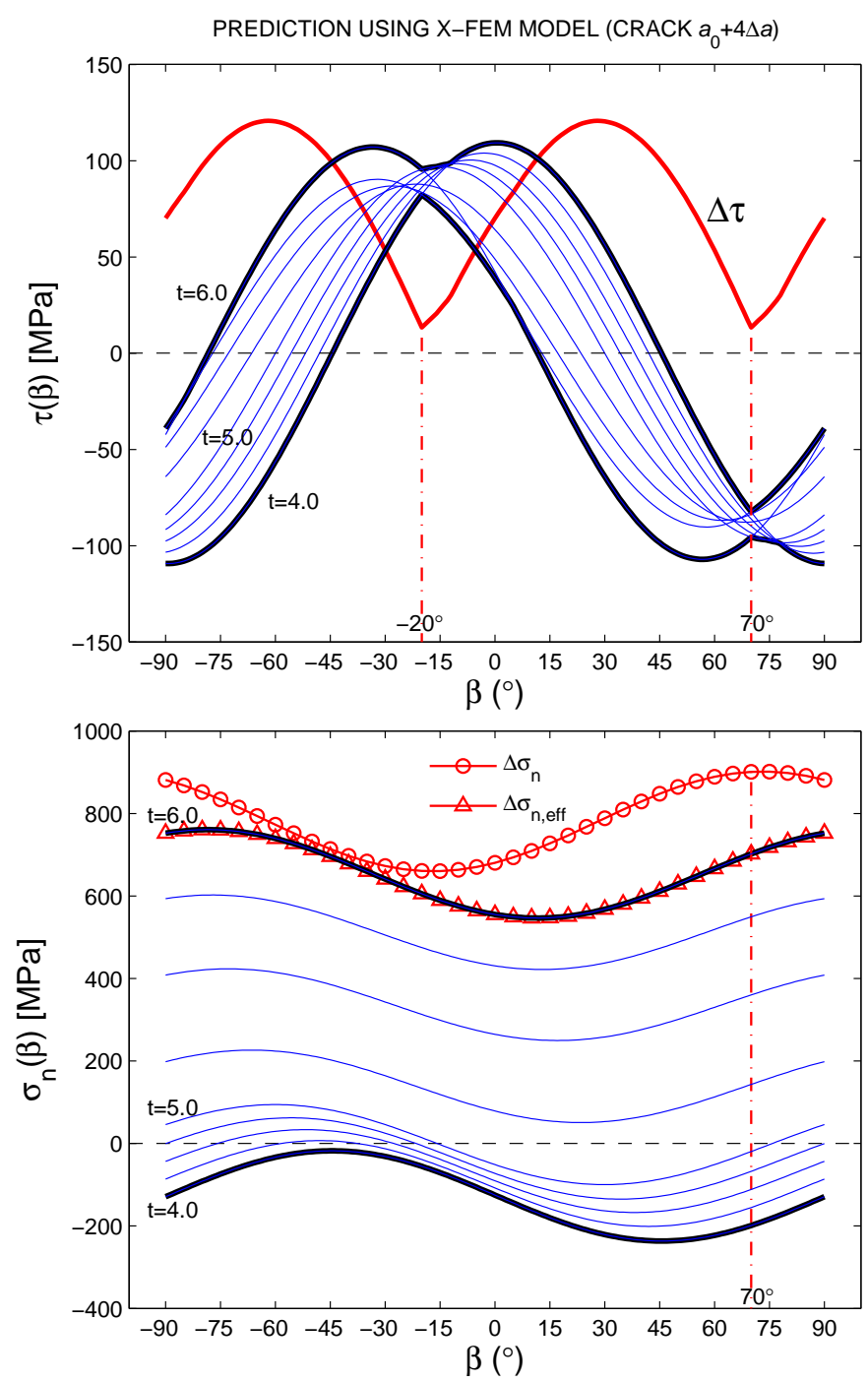

Figure 12: Prediction of crack growth direction ahead of the propagated crack after four crack growth increments, $a=a_{0}+4 \Delta a$, with the $\Delta \tau_{\min }$ criterion for test 1 . The crack is modelled using X-FEM.

Further examples of inaccurate growth orientations using the MTS criterion under non-proportional fretting loading can be found in Figs. 6 and 7 of one of our former works [27]. In that work, the MTS criterion was applied at the instant of maximum bulk load. All of our analyses predicted 

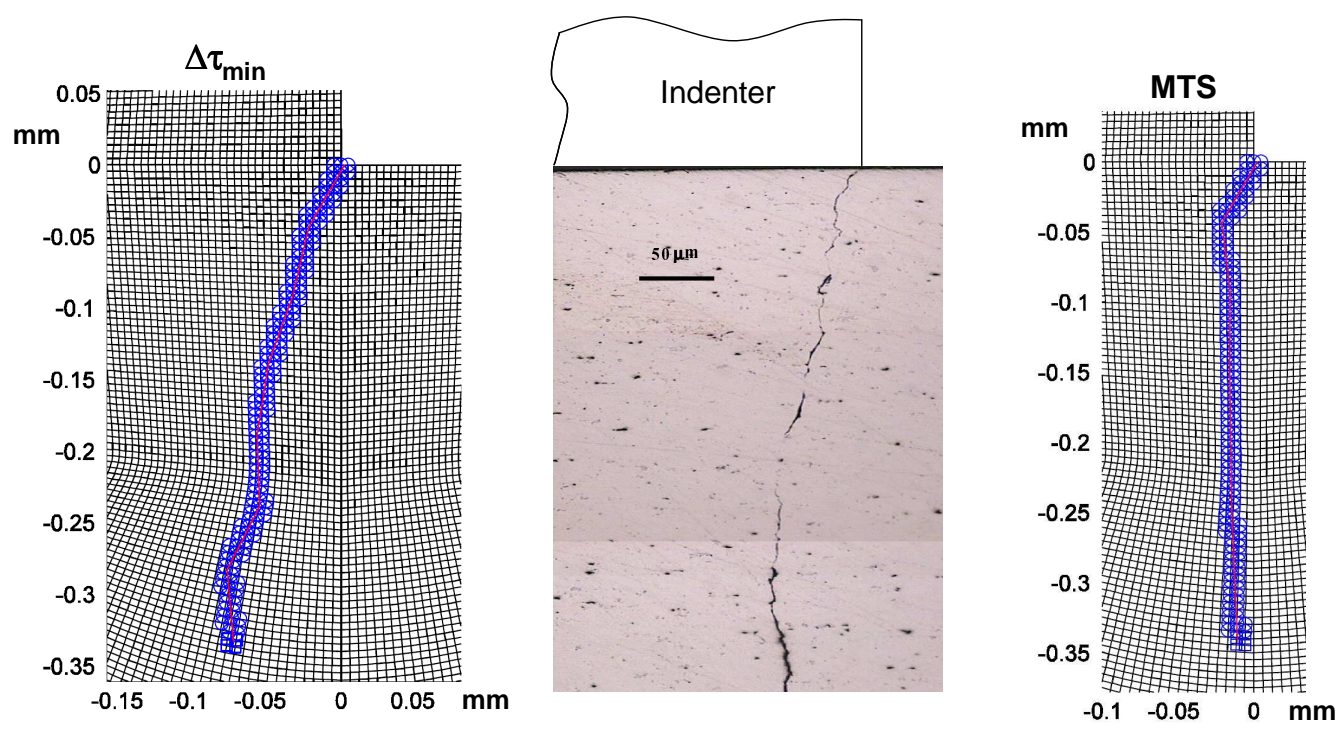

Figure 13: Predicted propagation path using X-FEM and the $\Delta \tau_{\min }$ vs. MTS criteria for test 1 .

growth paths outwards the contact zone, which is in contradiction with experimental evidence. In [32], the application of the MTS criterion to a fretting fatigue analysis at the instant of maximum load is also reported, yielding growth directions outwards the contact zone as well.

\subsection{Sensitivity to $\Delta a$ and the friction coefficient $\mu$}

Thanks to the versatility of the proposed procedure based on X-FEM, it is possible to study the influence of different parameters considered in the model. Fig. 14 (left) shows the influence of the assumed length $\Delta a$ for the crack propagation increment. It can be seen that the global trend is preserved, despite quite different values of $\Delta a$, thus proving the robustness of the procedure.

A more important issue from the physical viewpoint is the assumed value of the friction coefficient between crack faces. The above analyses have been 

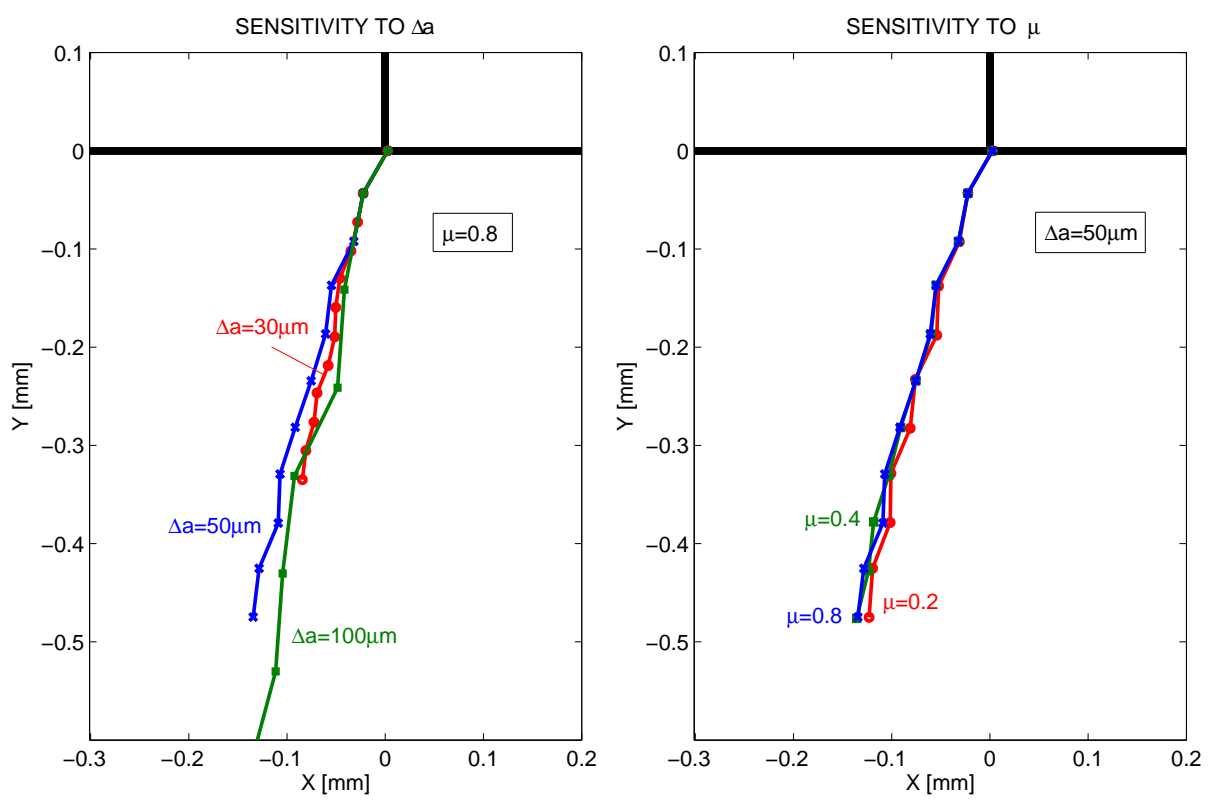

Figure 14: Sensitivity to different crack growth increments $\Delta a$ and friction coefficients $\mu$ for test 1 .

carried out with $\mu=0.8$ according to [31], but it is clear that the estimation of this parameter is elusive. Fig. 14 (right) shows that the influence of $\mu$ is not large. This is due to the kinks existing along the crack faces. The kinked segments prevent any sliding, blocking the crack faces after entering into contact. While this is obviously a numerical effect, it is presumed that a similar behaviour may happen in actual cracks due to the irregularities and asperities along the crack faces that can be observed in the micrographs of Figs. 5 and 10. Hence the right path can be predicted with the proposed procedure.

\section{Conclusions}

Crack propagation paths have been predicted for fretting fatigue tests under complete contact conditions. This type of problem is subjected to 
non-proportional loading, which invalidates the application of conventional orientation criteria usual in LEFM and that are only useful for proportional loading. To achieve these results, a criterion has been proposed based on the minimum value of $\Delta \tau$ evaluated ahead the crack tip and along the entire cycle. The prediction has been performed numerically using X-FEM including a formulation that allows for crack face contact, which is essential to take into account the effects during the compressive part of the cycle. The numerical results are in good agreement with the experimental observations.

\section{Acknowledgements}

The authors gratefully acknowledge the financial support given by the SGPI of the Spanish Ministry of Economics and Competitiveness (Projects DPI2007-66995-C03-02 and DPI2010-20990). The support of the Generalitat Valenciana, Programme PROMETEO 2012/023, is also acknowledged. The authors thank the collaboration of Mr. Pere Dasí Rodríguez.

\section{References}

[1] D.A. Hills, D. Nowell, Mechanics of fretting fatigue, Solid Mechanics and its Applications, Kluwer Academic Publishers, Dordrecht, 1994.

[2] E. Giner, M. Tur, A. Vercher, F.J. Fuenmayor, Numerical modelling of crack-contact interaction in 2D incomplete fretting contacts using X-FEM, Tribol. Int. 42 (2009) 1269-1275.

[3] E. Giner, C. Navarro, M. Sabsabi, M. Tur, J. Domínguez, F.J. Fuenmayor, Fretting fatigue life prediction using the extended finite element method, Int. J. Mech. Sci. 53 (2011) 217-225. 
[4] E. Giner, D.A. Hills, F.J. Fuenmayor, Complete elastic contact subject to cyclic shear in partial slip, J. Engng. Mech. - ASCE 131 (2005) 1146-1156.

[5] T. Hattori, M. Nakamura, T. Watanabe, Simulation of fretting-fatigue life by using stress-singularity parameters and fracture mechanics, Tribol. Int. 36 (2003) 87-97.

[6] M. Sabsabi, E. Giner, F.J. Fuenmayor, Experimental fatigue testing of a fretting complete contact and numerical life correlation using X-FEM, Int. J. Fatigue. 33 (2011) 811-822.

[7] E. Giner, N. Sukumar, J.E. Tarancón, F.J. Fuenmayor, An Abaqus implementation of the extended finite element method, Engng. Fract. Mech. 76 (2009) 347-368.

[8] E. Giner, M. Tur, J.E. Tarancón, F.J. Fuenmayor, Crack face contact in X-FEM using a segment-to-segment approach, Int. J. Numer. Methods Engng. 82 (2010) 1424-1449.

[9] M. Sabsabi, Modelado de grieta y estimación de vida en fretting fatiga mediante el método de los elementos finitos extendido X-FEM (in Spanish), PhD Thesis, Universitat Politècnica de València, Spain, 2010.

[10] V. Lamacq, M.C. Dubourg, L. Vincent, Crack path prediction under fretting fatigue - A theoretical and experimental approach, ASME J. Tribology 118 (1996) 711-720.

[11] P.J.E. Forsyth, A two stage process of fatigue crack growth, in: Proc. Crack Propagation Symposium, The College of Aeronautics, Cranfield, Vol. 1, 1961, pp. 76-94.

[12] M.C. Dubourg, V. Lamacq, Stage II crack propagation direction determination under fretting fatigue loading: a new approach in accordance with experimental observations, in: D.W. Hoeppner et al. (Eds.), Fretting Fatigue: Current Technology and Practices, ASTM STP 1367, West Conshohocken, 2000, pp. 436-450.

[13] A.F. Bower, The influence of crack face friction and trapped fluid on surface initiated rolling-contact fatigue cracks, ASME J Tribology 110 (1988) 704-711.

[14] P.E. Bold, M.W. Brown, R.J. Allen, A review of fatigue crack growth in steels under mixed mode I and II loading, Fatigue. Fract. Engng. Mater. Struct. 15 (1992) 965-977.

[15] F. Erdogan, G.C. Sih, On the crack extension path in plates under loading and transverse shear, J. Basic. Engng. 85 (1963) 519-527.

[16] G.C. Sih, Strain-energy-density factor applied to mixed mode crack problems, Int. 
J. Fracture. 10 (1974) 305-321.

[17] R. Ribeaucourt, M.C. Baietto-Dubourg, A. Gravouil, A new fatigue frictional contact crack propagation model with the coupled X-FEM/LATIN method, Comput. Methods Appl. Mech. Engng. 196 (2007) 3230-3247.

[18] F. Hourlier, A. Pineau, Fatigue crack path behavior under complex mode loading, in: D. François (Ed.), Advances in Fracture Research, Proc. 5th Int. Conference on Fracture, Pergamon, Oxford, 1981, pp. 1841-1849.

[19] M. Amestoy, H.-D. Bui, K. Dang Van, Déviation infinitésimale d'une fissure dans une direction arbitraire, Comptes Rendus Acad. Sci. Paris 289B (1979) 99-102.

[20] B. Cotterell, J.R. Rice, Slightly curved or kinked cracks, Int. J. Fracture. 16 (1980) $155-169$.

[21] T.L. Anderson, Fracture Mechanics: Fundamentals and Applications, 3rd ed., CRC Press, Boca Ratón, Florida, 2005.

[22] R.J. Nuismer, An energy release rate criterion for mixed mode fracture, Int. J. Fracture. 11 (1975) 245-250.

[23] E. Giner, M. Sabsabi, F.J. Fuenmayor, Calculation of $K_{\mathrm{II}}$ in crack face contacts using X-FEM. Application to fretting fatigue, Engng. Fract. Mech. 78(2) (2011) $428-445$.

[24] N. Moës, J. Dolbow, T. Belytschko, A finite element method for crack growth without remeshing, Int. J. Numer. Methods Engng. 46 (1999) 131-150.

[25] G. Zi, T. Belytschko, New crack-tip elements for XFEM and applications to cohesive cracks, Int. J. Numer. Methods Engng. 57 (2003) 2221-2240.

[26] G. Ventura, E. Budyn, T. Belytschko, Vector level sets for description of propagating cracks in finite elements, Int. J. Numer. Methods Engng. 58 (2003) 1571-1592.

[27] E. Giner, N. Sukumar, F.D. Denia, F.J. Fuenmayor, Extended finite element method for fretting fatigue crack propagation, Int. J. Solids Struct. 45 (2008) 5676-5687.

[28] S. Suresh, Fatigue of Materials, 2nd ed., Cambridge University Press, 1998.

[29] M.C. Baietto, E. Pierres, A. Gravouil, A multi-model X-FEM strategy dedicated to frictional crack growth under cyclic fretting fatigue loadings, Int. J. Solids Struct. 47 (2010) 1405-1423.

[30] E. Pierres, M.C. Baietto, A. Gravouil, G. Morales-Espejel, 3D two scale X-FEM 
crack model with interfacial frictional contact: Application to fretting fatigue, Tribol. Int. 43 (2010) 1831-1841.

[31] Y. Mutoh, J.Q. Xu, K. Kondoh, Observations and analysis of fretting fatigue crack initiation and propagation, in: Y. Mutoh, S.E. Kinyon, D.W. Hoeppner (Eds.), Fretting Fatigue: Advances in Basic Understanding and Applications, ASTM STP 1425, West Conshohocken, 2003, pp. 61-75.

[32] H.A. Fadag, S. Mall, V.K. Jain, A finite element analysis of fretting fatigue crack growth behaviour in Ti-6Al-4V, Engng. Fract. Mech. 75 (2008) 1384-1399. 